\title{
High risk of malnutrition is associated with low muscle mass in older hospitalized patients - a prospective cohort study
}

\author{
Vincent D. Pierik', Carel G. M. Meskers², Jeanine M. Van Ancum³, Siger T. Numans', Sjors Verlaan ${ }^{1,4}$,
} Kira Scheerman ${ }^{5}$, Roeliene C. Kruizinga ${ }^{1}$ and Andrea B. Maier ${ }^{3,6^{*}}$

\begin{abstract}
Background: Malnutrition, low muscle strength and muscle mass are highly prevalent in older hospitalized patients and associated with adverse outcomes. Malnutrition may be a risk factor for developing low muscle mass. We aimed to investigate the association between the risk of malnutrition and 1) muscle strength and muscle mass at admission and 2) the change of muscle strength and muscle mass during hospitalization in older patients.

Methods: The EMPOWER study included 378 patients aged seventy years or older who were acutely or electively admitted to four different wards of an academic teaching hospital in Amsterdam. Patients were grouped into low risk of malnutrition and high risk of malnutrition based on the Short Nutritional Assessment Questionnaire (SNAQ) score and were assessed for hand grip strength and muscle mass using hand held dynamometry respectively bioelectrical impedance analysis (BIA) within $48 \mathrm{~h}$ after admission and at day seven, or earlier at the day of discharge. Muscle mass was expressed as skeletal muscle mass, appendicular lean mass, fat free mass and the skeletal muscle index.

Results: The mean age of the patients was 79.7 years (SD 6.39), 48.9\% were female. At admission, being at high risk of malnutrition was significantly associated with lower muscle mass (Odds Ratio, 95\% Cl, 0.90, 0.85-0.96), but not with muscle strength. Muscle strength and muscle mass did not change significantly during hospitalization in both groups.

Conclusion: In older hospitalized patients, a high risk of malnutrition is associated with lower muscle mass at admission, but not with muscle strength nor with change of either muscle strength or muscle mass during hospitalization.
\end{abstract}

Keywords: Hospitalization, Muscle strength, Skeletal muscle, Malnutrition, Aged, Sarcopenia

\section{Background}

The physiological capacity often declines with age, making older patients vulnerable to the effects of hospitalization $[1,2]$. Physical inactivity during bed rest for injury or illness is a key feature during hospitalization [3]. Inactivity leads to alterations in protein synthesis and muscle breakdown which can result in loss of muscle mass, muscle strength and physical function $[4,5]$. Low muscle mass is

\footnotetext{
*Correspondence: a.b.maier@vu.nl

${ }^{3}$ Department of Human Movement Sciences, MOVE Research Institute

Amsterdam, VU University, Amsterdam, The Netherlands

${ }^{6}$ Department of Medicine and Aged Care, Royal Melbourne Hospital,

University of Melbourne, Melbourne, Australia

Full list of author information is available at the end of the article
}

independently associated with increased morbidity and mortality [6]. Ten percent of older patients suffer from sarcopenia (i.e. low muscle mass) at hospital admission [7]. The prevalence of sarcopenia can even increase due to illness and inactivity [8].

Malnutrition is an important risk factor for developing sarcopenia and is prevalent in $56 \%$ of patients on a geriatric ward $[6,9,10]$. Parameters of malnutrition were found to be associated with both relative and absolute muscle mass in geriatric outpatients [11]. The etiology of malnutrition in older patients is usually multifactorial and includes reduced nutritional intake and metabolic effects of illness [9]. Physiological changes also play an 
important role as older patients may suffer from anabolic resistance, resulting in a need for higher protein intake [4, 12]. In catabolic states, which often occur during malnutrition or acute illness, skeletal muscle is prone to muscle protein dissociation $[6,8]$. Malnutrition, particularly in combination with physical inactivity, may thus accelerate the process of sarcopenia which can result in serious adverse outcomes $[6,13,14]$. The extent to which malnutrition is related to muscle mass during hospitalization is not yet clear.

This study aimed to investigate the association between the risk of malnutrition, muscle strength and muscle mass at admission and change of muscle strength and muscle mass during hospitalization in older patients dependent on the risk of malnutrition.

\section{Methods}

\section{Design and patients}

The Evaluation of Muscle parameters in a Prospective cohort of Older patients at clinical Wards Exploring Relations with bed rest and malnutrition (EMPOWER) study is an observational, prospective, longitudinal inception cohort study. 838 Patients aged 70 years or older who were admitted to one of four clinical wards (acute admission, internal medicine, neurosurgery and orthopedics or traumatology) of the VU University Medical Center, Amsterdam, the Netherlands in the period from April 2015 to December 2015 were considered eligible and subsequently screened for participation in EMPOWER.

Patients had to sign informed consent to participate in this study. Patients were excluded if: (i) their expected length of stay was less than $24 \mathrm{~h}$; (ii) they were nursed in isolation rooms; (iii) they were terminally ill; (iv) they were not able to understand the Dutch language. Finally, 378 patients were included in the EMPOWER study (see Fig. 1). Patients were assessed at two occasions during their admission, i.e. within $48 \mathrm{~h}$ after admission, at the day of discharge or at day 7 after the first assessment if patients were still in hospital. If patients were discharged within $24 \mathrm{~h}$ after the first assessment, they were excluded from followup. 224 Patients (59\%) were assessed at two occasions during their admission. The study design was approved by the research ethics committee of the VU University Medical Center, Amsterdam, The Netherlands.

\section{Determinants and outcome measures}

Data collected from medical records included sociodemographics, number of medications, number of

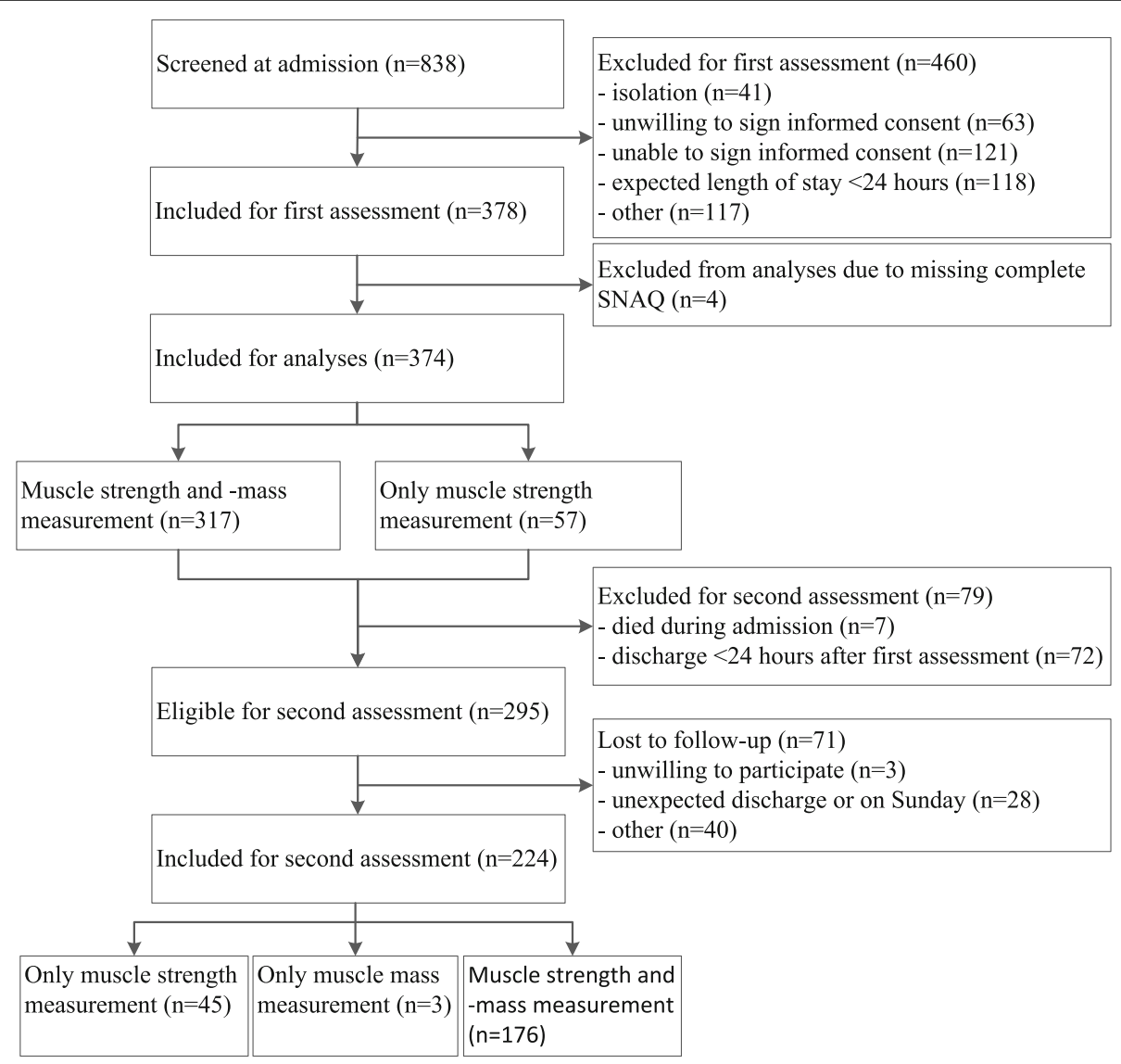

Fig. 1 Flowchart of patients included for each assessment 
comorbidities and attending medical specialism. Data that were gathered during an interview with the patient included alcohol use, smoking habits, use of a walking aid, living situation and falls during the last six months. Weight was measured on a weighing chair.

If patients were unable to get out of bed, an estimate was obtained from the patient or relative. Height was estimated using knee-height and the Longitudinal Aging Study Amsterdam formula (LASA): female: height $(\mathrm{cm})=68.74-(0.16 \mathrm{x}$ age $)+(2.07 \mathrm{x}$ knee-height in $\mathrm{cm})$, male: height $(\mathrm{cm})=74.48-(0.15 \mathrm{x}$ age $)+(2.03 \mathrm{x}$ kneeheight in $\mathrm{cm}$ ) [15]. Functional performance was assessed by the Katz index of independence in Activities of Daily Living (range 0-6) [16]. Cognition was scored with the 6-item cognitive impairment test (range $0-28$ ), a brief and simple test of cognition [17]. A numeric pain rating scale was used to assess pain in the patients (range 0-10) [18]. Functional ambulation classification was used to classify mobility (range 0-5) [19]. Brown's in-hospital mobility rating was used to rate physical activity during hospitalization (range 0-12) [20]. According to this rating; patients who walked at least two times a day were considered as a high-mobility group (median score $>8$ ).

\section{Risk of malnutrition}

Risk of malnutrition was determined using the Short Nutritional Assessment Questionnaire (SNAQ) score (range 0-7). The SNAQ is an easy, valid and reproducible questionnaire for early detection of hospital malnutrition including questions about unintentional weight loss, decrease in appetite over the last month and the use of supplemental drinks or tube feeding over the last month [21]. Four out of 378 patients were excluded from further analyses because of an incomplete SNAQ score. Patients were grouped into low risk of malnutrition (SNAQ-score 0-1) and high risk of malnutrition (SNAQ-score $\geq 2$ ). Screening for malnutrition by use of the SNAQ-score was part of regular care. Following the standard hospital care, patients at moderate risk of malnutrition (i.e. SNAQ-score 2) are offered energy- and protein rich meals and patients at severe risk of malnutrition (i.e. SNAQ-score > 2) are offered a dietary intervention. Including energy- and protein rich meals, supplementation or tube feeding.

\section{Muscle strength}

Muscle strength was measured by a Jamar dynamometer for hand grip strength (HGS) in a sitting position with elbows flexed at 90 degrees, shoulders adducted and forearms in neutral position without support. If patients were unable to get out of bed, HGS was measured with the bed in an angle of approximately 30 degrees and the elbows unsupported. Patients were actively encouraged to squeeze maximally. Both hands were assessed and two attempts were allowed per hand. The maximum score of either the left or the right hand was noted. At the second assessment patients were asked to take the same position as during the first assessment.

\section{Muscle mass parameters}

Muscle mass parameters were measured using a multi frequency bioelectrical impedance analyzer (BIA) (InBody S10, Biospace). Multi frequency BIA analysis is a valid tool for the assessment of body-composition and segmental lean measurements [22] and a good portable alternative to dual energy X-ray absorptiometry (DEXA), which is often used as a reference method [23]. Patients were asked to lie down in supine position with straightened arms and legs whenever able and to lie as still as possible during the measurement. Patients who were unable to lie down were measured in a sitting position with straightened arms and the BIA analyzer set to seated posture. Skeletal muscle mass, appendicular lean mass and fat free mass were noted. A distinction was made between absolute and relative values. Relative values of muscle-, appendicular leanand fat free mass were calculated by dividing the value by total body weight and multiplying it with $100 \%$. The skeletal muscle index was calculated by dividing skeletal muscle mass by squared height in meters [24]. In case of an implantable cardioverter defibrillator or other implanted devices $(n=29)$ or if it was impossible to position the electrodes at both middle fingers, thumbs and ankles $(n=28)$ BIA was not assessed.

\section{Statistical analyses}

To find a statistically significant difference of $0.5 \mathrm{~kg} / \mathrm{m}^{2}$ decrease in skeletal muscle index between two measurements with a power of $80 \%$ and an estimated standard deviation of 2.5 [25], 197 subjects had to be included.

Statistical Package for the Social Sciences (IBM SPSS Statistics for Windows, Version 23.0. Armonk, NY, IBM Corp) was used for analyses. Data with a skewed distribution were presented as median and interquartile range.

A logistic regression analysis was performed to analyze associations between the risk of malnutrition, muscle strength and muscle mass parameters (i.e. muscle mass, appendicular lean mass and fat free mass) at admission. Analyses were adjusted for age and sex (model 1) and additionally for comorbidities (model 2). To account for difference in body composition, absolute muscle mass parameters (skeletal muscle mass, appendicular lean mass and fat free mass) were additionally adjusted for relative muscle mass parameters at admission and relative muscle mass parameters (relative skeletal muscle mass, relative appendicular lean mass and relative fat free mass) for weight at admission (model 3). 
Paired samples t-tests were used to analyze changes of muscle strength and muscle mass parameters during hospitalization on significance in both groups. A logistic regression analysis was performed to analyze the associations between the risk of malnutrition, the change of muscle strength and muscle mass parameters during hospitalization. These analyses were adjusted for age, sex, time between the measurements and the corresponding value at admission (model 1 ). The other adjustment models were identical to the cross-sectional analyses. $P$-values below 0.05 were considered statistically significant.

\section{Results}

Table 1 shows the characteristics of the study population. The mean age was 79.7 years (SD 6.39) and $48.9 \%$ of the patients were female. At admission, $34.8 \%$ of the patients were at high risk of malnutrition according to the SNAQ (Fig. 2).

Table 2 shows the association of muscle strength and muscle mass parameters dependent on the risk of malnutrition at admission. The risk of malnutrition was not associated with muscle strength. High risk of malnutrition was significantly associated with lower absolute skeletal muscle-, appendicular lean- and fat free mass (Odds Ratios respectively $0.90,0.89$ and 0.95 ). None of the relative parameters of skeletal muscle-, appendicular lean- or fat free mass was associated with the risk of malnutrition.

Table 3 shows the changes of muscle strength and muscle mass parameters during hospitalization stratified by the risk of malnutrition. No significant changes of muscle strength, absolute muscle- or fat free mass were found. In both, the low-risk group and the high-risk group, absolute and relative appendicular lean mass increased significantly during hospital stay (respectively $0.8 \mathrm{~kg}$ and $1.4 \%$ ). Controlling the analyzes for volemic status based on clinical symptoms i.e. edema and skin turgor did not affect the results (data not shown). Relative fat free mass did increase significantly in the high-risk group (1.6\%). No differences in the change of muscle strength and muscle mass parameters during hospitalization were found dependent on the risk of malnutrition (Table 4).

Table 1 Patient characteristics of the entire cohort and stratified by the risk of malnutrition

\begin{tabular}{|c|c|c|c|c|}
\hline & \multirow[b]{2}{*}{$N$} & \multirow[b]{2}{*}{ All } & \multicolumn{2}{|c|}{ Risk of malnutrition } \\
\hline & & & Low & High \\
\hline & & & $n=244$ & $n=130$ \\
\hline Age, years, mean (sd) & 374 & $79.7(6.39)$ & $79.3(6.19)$ & $80.3(6.74)$ \\
\hline Sex, female & 374 & $183(48.9)$ & $123(50.4)$ & $60(46.2)$ \\
\hline Living independently & 373 & $327(87.7)$ & $217(88.9)$ & $110(85.3)$ \\
\hline Weight, kg, mean (sd) & 374 & $73.2(17.1)$ & $75.4(18.0)$ & $69.1(14.4)$ \\
\hline Height, cm, mean (sd) & 374 & $169(9.46)$ & $168(9.17)$ & 169 (9.99) \\
\hline $\mathrm{BMI}, \mathrm{kg} / \mathrm{m}^{2}$, mean $(\mathrm{sd})$ & 374 & $25.8(5.77)$ & $26.6(5.92)$ & $24.2(5.15)$ \\
\hline Current smoking & 365 & $39(10.7)$ & $29(12.2)$ & $10(7.8)$ \\
\hline Alcohol use & 364 & $146(40.1)$ & $112(47.5)$ & $34(26.6)$ \\
\hline Elective admission & 374 & $58(15.5)$ & $47(19.3)$ & $11(8.5)$ \\
\hline Admission, non-surgical & 374 & $205(54.8)$ & $115(47.1)$ & $90(69.2)$ \\
\hline Brown's-score $>8$ & 331 & $138(41,7)$ & $90(41,7)$ & $48(41,7)$ \\
\hline LOS, days, median (IQR) & 374 & $5.0(2.9-7.8)$ & $4.9(2.8-7.4)$ & $5.2(3.0-9.4)$ \\
\hline Number of medications $>4$ & 374 & $230(61.5)$ & $140(57.4)$ & $90(69.2)$ \\
\hline Number of comorbidities $>1$ & 372 & $329(88.4)$ & $214(88.1)$ & $115(88.5)$ \\
\hline KATZ ADL-score > 1 & 370 & $149(40.3)$ & $93(38.4)$ & $56(43.8)$ \\
\hline 6-item CIT, median (IQR) & 366 & $4(0-8)$ & $4(0-8)$ & $4(0-10)$ \\
\hline NRS-score on pain, median (IQR) & 370 & $2(0-5)$ & $2(0-6)$ & $1(0-5)$ \\
\hline FAC-score $>0$ & 370 & $273(73.8)$ & $166(68.9)$ & $107(82.9)$ \\
\hline Use of walking aid & 372 & $198(53.2)$ & $125(51.4)$ & $73(56.6)$ \\
\hline Fallen last six months & 374 & $169(45.2)$ & $118(48.4)$ & $51(39.2)$ \\
\hline Time between measurements, days, median (IQR) & 224 & $5.0(3.0-7.0)$ & $4.9(3.0-6.9)$ & $6.0(3.9-7.0)$ \\
\hline
\end{tabular}

All variables are presented as $n$ (\%) unless indicated otherwise. All variables were measured at baseline, except for length of stay and time between measurements

Brown's in-hospital mobility rating (range 0-12). LOS Length Of Stay. KATZ-ADL Katz Index of Independence in Activities of Daily Living (range 0-6). 6-item CIT 6-item Cognitive Impairment Test (range 0-28). NRS Numerical Rating Scale (range 0-10). FAC Functional Ambulation Classification (range 0-5) 


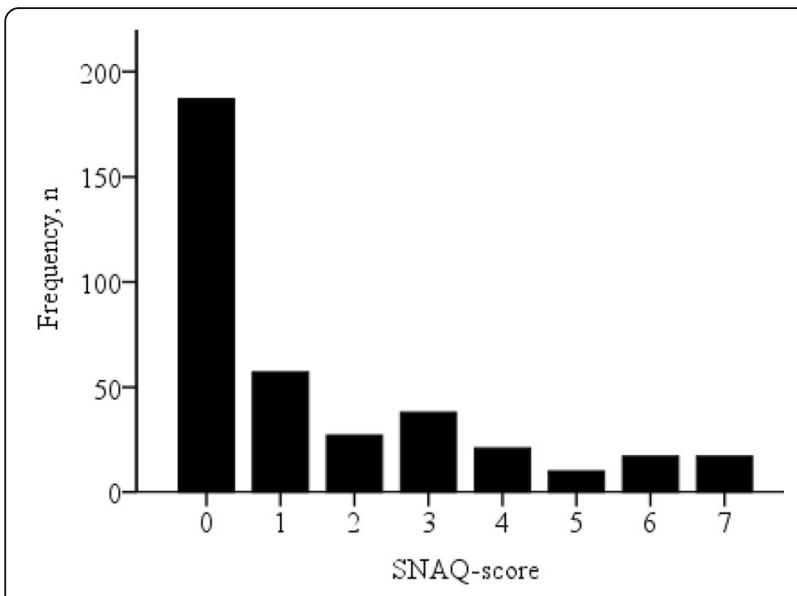

Fig. 2 Histogram of the Short Nutritional Assessment QuestionnaireScore of the Patients

\section{Discussion}

In this large inception cohort of older patients during hospitalization, a high risk of malnutrition was associated with lower muscle mass but not with lower muscle strength at admission. A change of muscle strength and muscle mass during hospitalization was not associated with the risk of malnutrition.

The association of a high risk of malnutrition and lower muscle mass at admission is in line with a crosssectional cohort study of 608 hospitalized patients with a significantly younger age compared to our cohort (median age 57 years) [26]. The prevalence of sarcopenia (i.e. low muscle mass) in that study was higher in patients that were grouped as moderately or severely malnourished based on the patient-generated subjective global assessment (PG-SGA).
To the best of our knowledge, no study has previously addressed the association between the risk of malnutrition at admission and change of muscle strength or muscle mass during hospitalization. We did expect to find a decrease of muscle mass during hospitalization due to the high prevalence of inactivity and malnutrition in older patient populations. A balance between anabolic and catabolic processes is required to maintain skeletal muscle mass [13]. Evidence shows that malnutrition can lead to a negative skeletal muscle protein balance, following muscle loss [4]. Theoretically, a week of physical inactivity increases skeletal muscle catabolism and decreases anabolism [27]. Notwithstanding, we did not find a significant decrease of skeletal muscle-, fat free mass and skeletal muscle index in the low-risk or the high-risk group. This was in line with a previous study in which no statistically significant change of fat free mass (measured by BIA) during hospitalization was found in 23 COPD patients with a mean age of 63 years [28]. In another study, a significant decrease of lean body mass was found after seven days of hospital stay in a group of 20 patients who had a median age of 70 years and underwent colorectal surgery [29]. This result may be due to low appetite, vomiting and disturbed gastrointestinal function after abdominal surgery in this selected patient population. Our study design minimized the risk of selection bias and the variety in specialisms ensured heterogeneity and a good representation of daily clinical practice.

Next to physical activity, nutrition is one of the main anabolic stimuli for muscle protein synthesis [30]. Muscle protein synthesis is driven by post-prandial plasma essential amino acid availability [27]. The patients who were at high risk of malnutrition and had a high protein intake as

Table 2 Hand grip strength and muscle mass parameters dependent on the risk of malnutrition

\begin{tabular}{|c|c|c|c|c|c|c|c|c|c|c|}
\hline & & & \multicolumn{2}{|c|}{ Risk of malnutrition } & \multicolumn{2}{|c|}{ Model 1} & \multicolumn{2}{|c|}{ Model 2} & \multicolumn{2}{|c|}{ Model 3} \\
\hline & & & Low & High & OR & $95 \% \mathrm{Cl}$ & OR & $95 \% \mathrm{Cl}$ & OR & $95 \% \mathrm{Cl}$ \\
\hline & & & $n=244$ & $n=130$ & & & & & & \\
\hline \multirow[t]{2}{*}{ HGS, kg } & $\hat{\sigma}$ & 191 & $27.1(10.3)$ & $24.3(8.91)$ & 0.98 & $0.95-1.01$ & 0.98 & $0.95-1.01$ & NA & \\
\hline & q & 183 & $15.1(5.75)$ & $14.8(5.35)$ & & & & & & \\
\hline SMM, kg & & 317 & $26.8(6.12)$ & $25.1(5.55)$ & 0.92 & $0.87-0.97$ & 0.92 & $0.86-0.97$ & 0.90 & $0.85-0.96$ \\
\hline ALM, kg & & 317 & $20.6(5.29)$ & $19.4(5.78)$ & 0.94 & $0.89-0.99$ & 0.94 & $0.88-0.99$ & 0.89 & $0.83-0.96$ \\
\hline FFM, kg & & 317 & $49.9(10.5)$ & $47.2(9.61)$ & 0.96 & $0.93-0.99$ & 0.95 & $0.92-0.99$ & 0.95 & $0.92-0.98$ \\
\hline \multirow[t]{2}{*}{$\mathrm{SMI}, \mathrm{kg} / \mathrm{m}^{2}$} & $\hat{\sigma}$ & 157 & $10.1(1.39)$ & $9.13(1.43)$ & 0.69 & $0.57-0.83$ & 0.68 & $0.56-0.83$ & NA & \\
\hline & q & 160 & $8.79(1.24)$ & $8.41(1.24)$ & & & & & & \\
\hline Relative SMM, \% & & 317 & $36.1(5.81)$ & $37.0(6.25)$ & 1.03 & $0.99-1.07$ & 1.03 & $0.98-1.07$ & 0.98 & $0.93-1.04$ \\
\hline Relative ALM, \% & & 317 & $27.6(4.74)$ & $28.6(7.37)$ & 1.03 & $0.99-1.09$ & 1.03 & $0.99-1.08$ & 1.01 & $0.96-1.06$ \\
\hline Relative FFM, \% & & 317 & $67.5(10.4)$ & $69.9(11.5)$ & 1.02 & $1.00-1.05$ & 1.02 & $1.00-1.05$ & 1.00 & $0.97-1.03$ \\
\hline
\end{tabular}

All variables are presented as mean (sd)

HGS Hand Grip Strength. SMM Skeletal Muscle Mass. ALM Appendicular Lean Mass. FFM Fat Free Mass. SMI Skeletal Muscle Index. NA Not Applicable. Model 1 adjusted for age and sex. Model 2 as 1 and comorbidities. Model 3 as 1 and absolute muscle parameters (SMM, ALM, FFM) for corresponding relative muscle parameter at admission, relative muscle parameters for weight at admission 
Table 3 Change of hand grip strength and muscle mass parameters stratified by the risk of malnutrition

\begin{tabular}{|c|c|c|c|c|c|c|c|c|}
\hline & & & \multicolumn{6}{|c|}{ Risk of malnutrition } \\
\hline & & & \multicolumn{3}{|c|}{ Low $n=159$} & \multicolumn{3}{|l|}{ High $n=65$} \\
\hline & & & Admission & In-hospital follow up & $P$ & Admission & In-hospital follow up & $P$ \\
\hline \multirow[t]{2}{*}{ HGS, kg } & 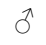 & 101 & $26.4(9.64)$ & $27.5(9.63)$ & 0.082 & $24.0(9.34)$ & $24.8(8.69)$ & 0.223 \\
\hline & q & 120 & $15.2(5.44)$ & $15.9(5.24)$ & 0.069 & $13.0(4.65)$ & $14.2(4.94)$ & 0.115 \\
\hline SMM, kg & & 179 & $25.7(5.36)$ & $25.6(5.47)$ & 0.455 & $24.5(5.75)$ & $24.8(5.60)$ & 0.270 \\
\hline ALM, kg & & 179 & $19.6(4.77)$ & $20.0(4.87)$ & 0.030 & $18.7(5.19)$ & $19.5(5.30)$ & 0.007 \\
\hline FFM, kg & & 179 & $48.1(9.20)$ & $48.2(9.43)$ & 0.678 & $46.2(10.0)$ & $47.1(9.96)$ & 0.088 \\
\hline \multirow[t]{2}{*}{$\mathrm{SMI}, \mathrm{kg} / \mathrm{m}^{2}$} & $\widehat{o}$ & 78 & $9.90(1.21)$ & $9.83(1.23)$ & 0.404 & $9.00(1.65)$ & $9.09(1.65)$ & 0.470 \\
\hline & q & 101 & $8.67(1.18)$ & $8.62(1.33)$ & 0.683 & $8.46(1.27)$ & $8.58(1.07)$ & 0.411 \\
\hline Relative SMM, \% & & 179 & $36.6(5.59)$ & $36.3(5.62)$ & 0.380 & $36.8(6.73)$ & $37.4(7.24)$ & 0.128 \\
\hline Relative ALM, \% & & 179 & $27.6(4.37)$ & $28.2(4.82)$ & 0.027 & $27.9(5.54)$ & $29.3(6.81)$ & 0.002 \\
\hline Relative FFM, \% & & 179 & $68.5(10.1)$ & $68.6(10.3)$ & 0.800 & $69.7(12.6)$ & $71.3(13.8)$ & 0.038 \\
\hline
\end{tabular}

All variables are presented as mean (sd). HGS Hand Grip Strength. SMM Skeletal Muscle Mass. ALM Appendicular Lean Mass. FFM Fat Free Mass. SMI Skeletal Muscle Index

a result of the standard hospital care, may have had a higher muscle protein synthesis. A randomized-controlled study in 592 acutely ill older patients, not selecting on nutritional risk groups, showed that there were no significant differences in change of mid-arm circumference, triceps skinfold thickness and hand grip strength between the intervention group with additional nutritional care and the standard care group [31]. Another study in 23 hospitalized malnourished elderly patients showed a positive effect on fat free mass assessed by DXA, but not on hand grip strength, after ten days of dietary supplementation [32].

BIA measurements are relatively easy to perform with minimal burden and therefore well suited to measure body composition in vulnerable older patients. However, use of BIA may have some drawbacks as it could have been influenced by the hydration status of older patients. BIA estimates body composition by the difference in impedance of various tissues. Adipose tissue contains a relatively low amount of water compared to muscle tissue and therefore has higher impedance. A previous study of 200 acutely admitted older patients found a high prevalence of dehydration at hospital admission, which decreased during hospitalization [33]. In the same study the prevalence of malnutrition, based on the nutritional risk screening (NRS 2002), did not differ between the euhydrated and the dehydrated group. Taken together, fat tissue may have been overestimated during the measurements at admission, resulting in lower appendicular lean- and fat free mass in both the low-risk

Table 4 Differences in change of hand grip strength and muscle mass parameters dependent on the risk of malnutrition

\begin{tabular}{|c|c|c|c|c|c|c|c|c|c|c|}
\hline & & & \multicolumn{2}{|c|}{ Risk of malnutrition } & \multicolumn{2}{|c|}{ Model 1} & \multicolumn{2}{|c|}{ Model 2} & \multicolumn{2}{|c|}{ Model 3} \\
\hline & & & Low & High & $\mathrm{OR}$ & $95 \% \mathrm{Cl}$ & OR & $95 \% \mathrm{Cl}$ & OR & $95 \% \mathrm{Cl}$ \\
\hline & & & $n=159$ & $n=65$ & & & & & & \\
\hline \multirow[t]{2}{*}{ HGS, kg } & $\hat{\sigma}$ & 101 & $+1.05(4.77)$ & $+0.75(3.62)$ & 1.01 & $0.93-1.09$ & 1.01 & $0.93-1.09$ & NA & \\
\hline & q & 120 & $+0.78(4.00)$ & $+1.23(4.20)$ & & & & & & \\
\hline SMM, kg & & 179 & $-0.14(2.09)$ & $+0.28(1.86)$ & 1.05 & $0.89-1.23$ & 1.05 & $0.89-1.23$ & 1.05 & $0.89-1.24$ \\
\hline ALM, kg & & 179 & $+0.38(1.93)$ & $+0.80(2.08)$ & 1.05 & $0.88-1.24$ & 1.05 & $0.88-1.24$ & 1.05 & $0.88-1.24$ \\
\hline FFM, kg & & 179 & $+0.14(3.81)$ & $+0.85(3.55)$ & 1.02 & $0.93-1.12$ & 1.02 & $0.93-1.12$ & 1.03 & $0.94-1.12$ \\
\hline \multirow[t]{2}{*}{$\mathrm{SMl}, \mathrm{kg} / \mathrm{m}^{2}$} & $\delta$ & 78 & $-0.06(0.56)$ & $+0.09(0.61)$ & 1.06 & $0.68-1.66$ & 1.06 & $0.68-1.66$ & NA & \\
\hline & q & 101 & $-0.04(0.92)$ & $+0.12(0.76)$ & & & & & & \\
\hline Relative SMM, \% & & 179 & $-0.25(3.16)$ & $+0.60(2.84)$ & 1.07 & $0.96-1.19$ & 1.07 & $0.96-1.19$ & 1.06 & $0.95-1.18$ \\
\hline Relative ALM, \% & & 179 & $+0.58(2.88)$ & $+1.44(3.24)$ & 1.06 & $0.95-1.19$ & 1.06 & $0.95-1.19$ & 1.06 & $0.94-1.18$ \\
\hline Relative FFM, \% & & 179 & $+0.13(5.73)$ & $+1.58(5.39)$ & 1.03 & $0.98-1.10$ & 1.03 & $0.98-1.10$ & 1.03 & $0.97-1.09$ \\
\hline
\end{tabular}

All variables are presented as mean (sd). HGS Hand Grip Strength. SMM Skeletal Muscle Mass. ALM Appendicular Lean Mass. FFM Fat Free Mass. SMI Skeletal Muscle Index. NA Not Applicable. Model 1 adjusted for age, sex, time between the two measurements and value at admission. Model 2 as 1 and comorbidities. Model 3 as 1 and absolute muscle parameters (SMM, ALM, FFM) for corresponding relative muscle parameter at admission, relative muscle parameters for weight at admission 
and the high-risk group. The increase of appendicular lean mass could be explained by this phenomenon. A decrease of fat free mass may have been masked in both groups.

This study included 378 participants at admission and ended up with 224 participants, of whom 179 participants with two measurements of muscle mass parameters. This implies that the study was slightly underpowered. Nevertheless, this study is the biggest until now reporting follow up data [34] and the standard deviation of skeletal muscle index was much smaller compared to the previous study [25], giving this study enough power to draw conclusions.

\section{Strengths and limitations}

The large inception cohort of a relevant group of patients who were acute or elective admitted to different wards of surgical and non-surgical specialisms ensured heterogeneity in this study. However, the observational design did not allow us to draw conclusions concerning possible interventions. Furthermore, the use of BIA instead of magnetic resonance imaging (MRI) or computed tomography (CT) scan and the standard hospital care for patients at risk of malnutrition may have influenced our results.

\section{Conclusion}

In older hospitalized patients, a high risk of malnutrition was significantly associated with lower absolute skeletal muscle-, appendicular lean-, fat free mass and the skeletal muscle index, but not with lower muscle strength. The risk of malnutrition was not associated with a change of these parameters during hospitalization. Further research is needed to determine the long-term impact of hospitalization on muscle mass in older patients.

\section{Abbreviations}

6-item CIT: 6-item Cognitive impairment test; ALM: Appendicular lean mass; BIA: Bioelectrical impedance analysis; DEXA: Dual energy x-ray absorptiometry; EMPOWER: The evaluation of muscle parameters in a prospective cohort of older patients at clinical Wards Exploring Relations with bed rest and malnutrition; FAC: Functional ambulation classification; FFM: Fat free mass; HGS: Hand grip strength; KATZ-ADL: Katz index of independence in activities of daily living; NRS: Numerical rating scale; SMI: Skeletal muscle index; SMM: Skeletal muscle mass; SNAQ: Short Nutritional Assessment Questionnaire

\section{Acknowledgements}

The authors would like to thank A. Verburg and H.M.D. Nagtzaam for their assistance during the inclusion of patients and Monique Slee-Valentijn for the help in designing the protocol.

\section{Funding}

This study was financially supported by Nutricia Research, Nutricia Advanced Medical Nutrition, The Netherlands.

\section{Availability of data and materials}

The datasets analyzed during the current study are available from the corresponding author on reasonable request.

\section{Authors' contributions}

Conceived the study protocol and design: SV, RCK, CGMM and ABM. Collected data: VDP, JMvA, STN, KS and RCK. Analyzed the data: VDP, JMvA and STN. Contributed to analyses: JMvA, STN, SV, KS, CGMM and ABM. Drafted the article: VDP. Critically revised the article: CGMM, JMVA, STN, SV $\mathrm{KS}, \mathrm{RCK}$ and $\mathrm{ABM}$. All authors read and approved the final manuscript.

\section{Competing interests}

The authors declare that they have no conflicts of interest. Sjors Verlaan is an employee at Nurticia Research, Nutricia Advanced Medical Nutrition.

Consent for publication

Not applicable.

Ethics approval and consent to participate

The study design was approved by the research ethics committee of the VU University Medical Center, Amsterdam, The Netherlands. Written informed consent was obtained from all participants.

\section{Publisher's Note}

Springer Nature remains neutral with regard to jurisdictional claims in published maps and institutional affiliations.

\section{Author details}

'Department of Internal Medicine, Section of Gerontology and Geriatrics, VU University Medical Center, Amsterdam, The Netherlands. ${ }^{2}$ Department of Rehabilitation Medicine, VU University Medical Center, Vrije Universiteit De Boelelaan 1105, 1081HV Amsterdam, The Netherlands. ${ }^{3}$ Department of Human Movement Sciences, MOVE Research Institute Amsterdam, VU University, Amsterdam, The Netherlands. ${ }^{4}$ Nutricia Research, Nutricia Advanced Medical Nutrition, Utrecht, The Netherlands. ${ }^{5}$ Division I, VU University Medical Center, Amsterdam, The Netherlands. ${ }^{6}$ Department of Medicine and Aged Care, Royal Melbourne Hospital, University of Melbourne, Melbourne, Australia.

Received: 27 September 2016 Accepted: 22 May 2017

Published online: 05 June 2017

\section{References}

1. Mudge AM, O'Rourke P, Denaro CP. Timing and risk factors for functional changes associated with medical hospitalization in older patients. J GerontolSer A Biol Sci Med Sci. 2010;65 A(8):866-72. doi:10.1093/gerona/glq069.

2. Kortebein $P$, Symons TB, Ferrando A, et al. Functional impact of 10 days of bed rest in healthy older adults. J Gerontol A Biol Sci Med Sci. 2008; 63(10):1076-81.

3. Brown CJ, Redden DT, Flood KL, et al. The underrecognized epidemic of low mobility during hospitalization of older adults. J Am Geriatr Soc. 2009; 57(9):1660-5. doi:10.1111/j.1532-5415.2009.02393.x.

4. Deutz NEP, Bauer JM, Barazzoni $R$, et al. Protein intake and exercise for optimal muscle function with aging: recommendations from the ESPEN expert group. Clin Nutr. 2014;33(6):929-36. doi:10.1016/j.clnu.2014.04.007.

5. Coker $\mathrm{RH}$, Hays NP, Williams $\mathrm{RH}$, et al. Bed rest promotes reductions in walking speed, functional parameters, and aerobic fitness in older, healthy adults. J Gerontol A Biol Sci Med Sci. 2014;70(1):1-6. doi:10. 1093/gerona/glu123.

6. Vandewoude MFJ, Alish CJ, Sauer AC, et al. Malnutrition-sarcopenia syndrome: Is this the future of nutrition screening and assessment for older adults? J Aging Res. 2012; 2012. doi:10.1155/2012/651570.

7. Gariballa S, Alessa A. Sarcopenia: Prevalence and prognostic significance in hospitalized patients. Clin Nutr. 2013;32(5):772-6. doi:10.1016/j.clnu.2013.01.010.

8. Evans WJ, Paolisso G, Abbatecola AM, et al. Frailty and muscle metabolism dysregulation in the elderly. Biogerontology. 2010;11(5):527-36. doi:10.1007/ s10522-010-9297-0.

9. Pirlich M, Schütz T, Norman K, et al. The German hospital malnutrition study Clin Nutr. 2006;25(4):563-72. doi:10.1016/j.clnu.2006.03.005.

10. Edington J, Boorman J, Durrant ER, et al. Prevalence of malnutrition on admission to four hospitals in England. The Malnutrition Prevalence Group. Clin Nutr. 2000;19(3):191-5. doi:10.1054/clnu.1999.0121.

11. Reijnierse EM, Trappenburg MC, Leter MJ, et al. The association between parameters of malnutrition and diagnostic measures of sarcopenia in geriatric outpatients. PLoS One. 2015;10(8):e0135933. doi:10.1371/journal. pone.0135933. 
12. Tieland M, Borgonjen-Van den Berg K, Van Loon L, et al. Dietary protein intake in Dutch elderly people: a focus on protein sources. Nutrients. 2015; 7(12):9697-706. doi:10.3390/nu7125496.

13. Lang T, Streeper T, Cawthon P, et al. Sarcopenia: etiology, clinical consequences, intervention, and assessment. Osteoporos Int. 2010;21(4): 543-59. doi:10.1007/s00198-009-1059-y.

14. Cruz-Jentoft AJ, Landi F, Topinková E, et al. Understanding sarcopenia as a geriatric syndrome. Curr Opin Clin Nutr Metab Care. 2010;13(1):1-7. doi:10. 1097/MCO.0b013e328333c1c1.

15. LASA. http://www.lasa-vu.nl/themes/physical/recoding-height-and-weight. htm . Accessed 4 Jan 2016

16. Reijneveld SA, Spijker J, Dijkshoorn H. Katz' ADL index assessed functional performance of Turkish, Moroccan, and Dutch elderly. J Clin Epidemiol. 2007;60(4):382-8. doi:10.1016/j.jclinepi.2006.02.022.

17. Upadhyaya KA, Rajagopal M, Gale MT. The six item cognitive impairment test (6-CIT) as a screening test for dementia: comparison with mini-mental state examination (MMSE). Curr Aging Sci. 2010;3(2):138. http://www. ingentaconnect.com/content/ben/cas/2010/00000003/00000002/art00006. Accessed 16 Oct 2015

18. Williamson A, Hoggart B. Pain: a review of three commonly used pain rating scales. J Clin Nurs. 2005;14(7):798-804. doi:10.1111/j.1365-2702.2005.01121.x.

19. Holden MK, Gill KM, Magliozzi MR, et al. Clinical Gait Assessment in the Neurologically Impaired Reliability and Meaningfulness. Phys Ther. 1984; 64(1):35-40.

20. Brown C, Friedkin R, Inouye S. Prevalence and outcomes of low mobility in hospitalized older patients. J Am Geriatr Soc. 2004;52(8):1263-70.

21. Kruizenga HM, Seidell JC, de Vet HCW, et al. Development and validation of a hospital screening tool for malnutrition: the short nutritional assessment questionnaire (SNAQC). Clin Nutr. 2005;24(1):75-82. doi:10.1016/j.clnu.2004.07.015.

22. Ling CHY, de Craen AJM, Slagboom PE, et al. Accuracy of direct segmental multi-frequency bioimpedance analysis in the assessment of total body and segmental body composition in middle-aged adult population. Clin Nutr. 2011;30(5):610-5. doi:10.1016/j.clnu.2011.04.001.

23. Cruz-Jentoft AJ, Baeyens JP, Bauer JM, et al. Sarcopenia: European consensus on definition and diagnosis. Age Ageing. 2010;39(4):412-23. doi: 10.1093/ageing/afq034.

24. Janssen I, Baumgartner RN, Ross R, et al. Skeletal muscle Cutpoints associated with elevated physical disability risk in older men and women. Am J Epidemiol. 2004;159(4):413-21. doi:10.1093/aje/kwh058.

25. Bianchi L, Savino E, Magon S, et al. Loss of muscle mass in older patients hospitalized in acute care wards: results from the CRIME study. Europ Ger Med. 2013;4:p46.

26. Sousa AS, Guerra RS, Fonseca I, et al. Sarcopenia among hospitalized patients - a cross-sectional study. Clin Nutr. 2015;34(6):1-6. doi:10.1016/j. clnu.2014.12.015

27. Wall BT, Dirks ML, van Loon LJC. Skeletal muscle atrophy during short-term disuse: implications for age-related sarcopenia. Ageing Res Rev. 2013;12(4): 898-906. doi:10.1016/j.arr.2013.07.003.

28. Vermeeren MA, Schols AMWJ, Wouters EFM. Effects of an acute exacerbation on nutritional and metabolic profile of patients with COPD. Eur Respir J. 1997;10(10):2264-9. doi:10.1183/09031936.97.10102264.

29. Henriksen MG, Hansen HV, Hessov I. Early oral nutrition after elective colorectal surgery: influence of balanced analgesia and enforced mobilization. Nutrition. 2002;18:263-7. doi:10.1016/S0899-9007(01)00749-3.

30. Koopman R, van Loon LJC. Regulation of protein metabolism in exercise and recovery: aging, exercise, and muscle protein metabolism. J Appl Physiol. 2009;106:2040-8. doi:10.1152/japplphysiol.91551.2008.

31. Hickson M, Bulpitt C, Nunes M, et al. Does additional feeding support provided by health care assistants improve nutritional status and outcome in acutely ill older in-patients? - a randomised control trial. Clin Nutr. 2004; 23(1):69-77. doi:10.1016/50261-5614(03)00090-6.

32. Bos C, Benamouzig R. Bruhat a, et al. nutritional status after short-term dietary supplementation in hospitalized malnourished geriatric patients. Clin Nutr. 2001;20(3):225-33. doi:10.1054/clnu.2000.0387.

33. El-Sharkawy A, Watson P, Neal K, et al. Hydration and outcome in older patients admitted to hospital (The HOOP prospective cohort study). Age Ageing. 2015;(August):1-5. doi:10.1093/ageing/afv119..

34. Ancum JM Van, Scheerman $\mathrm{K}$, Jonkman $\mathrm{NH}$, et al. Change in muscle strength and muscle mass in older hospitalized patients: A systematic review and meta-analysis. Exp Gerontol. 2017;92:34-41.

\section{Submit your next manuscript to BioMed Central and we will help you at every step:}

- We accept pre-submission inquiries

- Our selector tool helps you to find the most relevant journal

- We provide round the clock customer support

- Convenient online submission

- Thorough peer review

- Inclusion in PubMed and all major indexing services

- Maximum visibility for your research

Submit your manuscript at www.biomedcentral.com/submit 